\title{
Progressive Distributed Coding of Multispectral Images
}

\author{
Jinrong Zhang \\ Univ. of Science and Tech. of China \\ Hefei, 230027, P. R. China \\ $+86-551-3601340$ \\ jinrong@mail.ustc.edu.cn
}

\author{
Houqiang Li \\ Univ. of Science and Tech. of China \\ Hefei, 230027, P. R. China \\ $+86-551-3601340$ \\ lihq@ustc.edu.cn
}

\author{
Chang Wen Chen \\ State Univ. of New York at Buffalo \\ Buffalo, NY, 14260, USA \\ $+1-716-645-3180$ \\ chencw@buffalo.edu
}

\begin{abstract}
We present in this paper a novel distributed coding scheme for lossless and progressive compression of multispectral images. The main strategy of this new scheme is to explore data redundancies at the decoder in order to design a lightweight yet very efficient encoder suitable for onboard applications during acquisition of multispectral image. A sequence of increasing resolution layers is encoded and transmitted successively until the original image can be losslessly reconstructed from all layers. We assume that the decoder with abundant resources is able to perform adaptive region-based predictor estimation to capture spatially varying spectral correlation with the knowledge of lower-resolution layers, thus generate high quality side information for decoding the higher-resolution layer. Progressive transmission enables the spectral correlation to be refined successively, resulting in gradually improved decoding performance of higher-resolution layers as more data are decoded. Simulations have been carried out to demonstrate that the proposed scheme, with innovative combination of low complexity encoding, lossless compression and progressive coding, can achieve competitive performance comparing with high complexity state-of-the-art 3-D DPCM technique.
\end{abstract}

\section{Categories and Subject Descriptors}

E.4 [Coding and Information Theory]: Data Compaction and Compression - image compression, distributed coding

\section{General Terms}

Algorithms/Design

\section{Keywords}

Distributed coding, progressive coding, lossless compression, multispectral images, low complexity encoding

\section{INTRODUCTION}

Multispectral images are composed of a number of bands

Permission to make digital or hard copies of all or part of this work for personal or classroom use is granted without fee provided that copies are not made or distributed for profit or commercial advantage and that copies bear this notice and the full citation on the first page. To copy otherwise, or republish, to post on servers or to redistribute to lists, requires prior specific permission and/or a fee.

Mobimediae'09, September 7-9, 2009, London, United Kingdom

Copyright 2009 ICST 978-963-9799-62-2/00/0004_..\$5.00 representing the same area of the earth surface in different spectral intervals. As the contemporary technologies advance in remote sensing systems, multispectral data are produced at increasingly higher spatial, spectral, temporal and radiometric resolutions. However, together with this wealth of information generation comes the challenges of transmitting such huge amount of data over limited-capacity channels from the remote platform to the ground station. Thus, efficient compression algorithms are highly demanded in order to match the available bandwidth to the ever increasing multispectral image data generation. Since even the least distortion may lead to erroneous interpretation of the considered scene or incorrect estimation of the ground parameters, we focus our research on lossless compression in order to recover the original image from the compressed data without any loss of information.

Another desirable feature for multispectral images compression is progressive reconstruction. Such feature is very useful when users are browsing the image data for specific applications. This requires the encoder to produce a bit stream that is able to provide a sequence of resolution layers for effective delivery. The first layer corresponds to some highly compressed version and each successive layer provides more details. These layers are decoded successively so that the image is quickly displayed at low-resolution and then gradually refined. For the area in which users have little interest, the corresponding high-resolution layers need not be transmitted so that bandwidth can be saved for transmitting more useful image data. The recent still-image compression standard, JPEG 2000, provides such features in its extended form [1]. However, like other two-dimensional (2-D) schemes [2], JPEG 2000 is unable to obtain optimal results for the inherently threedimensional (3-D) multispectral images since only spatial redundancy is exploited. To improve compression performance, some 3-D schemes have been specifically designed in the last few years for multispectral images in order to efficiently remove both spatial redundancy and spectral redundancy [3], [4], [5], [6]. Although these 3-D schemes can provide high compression performance and some of them offer progressive transmission [3], [4], the data decorrelation implemented at the encoder, i.e., prediction or discrete wavelet transform (DWT), are generally computation sensitive and memory consumptive. This will impose on the encoder a heavy computational burden that a remote platform is usually unable to afford. Therefore, the remote sensing systems have been longing for a completely new coding scheme with competing requirements in low computational complexity at encoder and high compression efficiency for image coding. The objective of this research is indeed to develop a scheme to meet such challenges and to provide progressive compression feature aiming at the required onboard applications. 


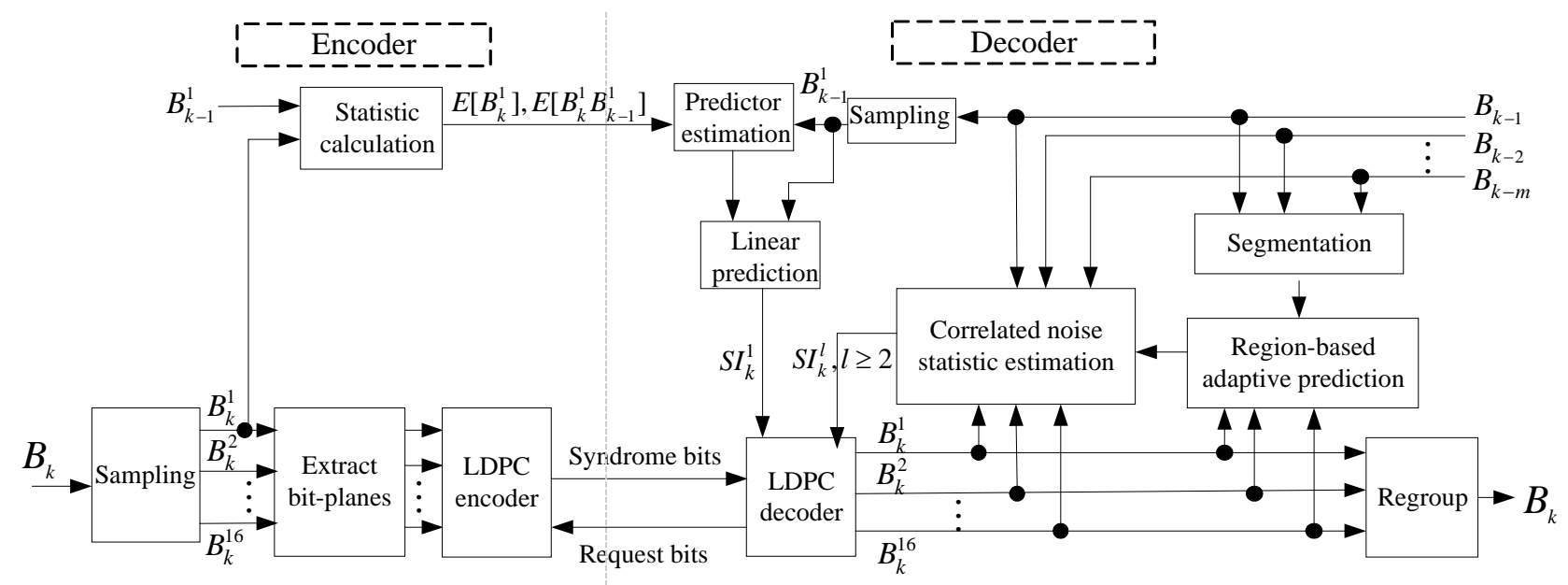

Figure 1: Framework of the proposed system

To design a low complexity encoder, we need to move the high complexity exploitation of data correlation to the decoder on the ground, where computation capability and memory capacity are abundant. Distributed source coding (DSC) provides a new and promising solution to such a problem. Based on Slepian-Wolf theory [7], correlated sources can be compressed by separate encoders without performance loss as long as they are jointly decoded. This allows us to move computation complexity from encoder to decoder so as to facilitate the resources allocation of remote sensing systems. However, there has been surprisingly little work on DSC of multispectral images. Until recently, only a few approaches have been attempted to assess the potential of DSC for multispectral image compression [8], [9]. Over the years, the straightforward spectral decorrelation methods have led to some unacceptable performance loss, and the spatial decorrelation operations via DWT/spatial prediction, have increased encoder's computation burden and resulted in very limited practical schemes.

In this research, we seek to design a practical DSC-based codec with the desired characteristics of progressive transmission, low complexity encoding, lossless compression and high compression efficiency suitable for onboard applications. The image is first partitioned into a number of slices by specially designed sampling. These slices are then encoded independently and transmitted progressively. Decoding starts from the first slice and the lowestresolution layer is then reconstructed. As more slices are decoded, a higher-resolution layer can be obtained. Ultimately, the original image is completely recovered after all slices are decoded. Although the first slice contains significantly reduced data, it is still adequate to represent the spectral dependency of the original image since spatially adjacent pixels retain similar spectral dependency. The subsequent slices are successively decoded with the side information generated by accessing the previously decoded slices as well as the preceding multispectral bands. To capture the nonstationary spectral correlation of multispectral images, an adaptive region-based prediction algorithm is performed to acquire high quality side information. With more slices available, the decoder acquires higher quality side information and thus better compression performance. Comparing with the conventional entropy coding based 3-D schemes [3], [4], [5], [6], the proposed DSC approach has an additional advantage besides low encoding complexity and progressive transmission. That is, it can also provide potential error resilience by preventing error propagation caused by prediction mismatch which often occurs in predictive coding schemes. Furthermore, as decorrelation transform is not employed, the proposed scheme is easy to be extended to near-lossless compression application by adding a quantizer before extracting bit-planes so as to provide higher compression ratio. The reason that a transform coding scheme is not suitable for near-lossless compression has been discussed in [6].

It should be noted that to the best of our knowledge, this is the first distributed coding-based scheme that provides lossless as well as progressive reconstruction for multispectral images. The compression performance of the proposed scheme is comparable to the best-known lossless compression technique reported in the literature with much reduced encoder complexity.

The rest of this paper is organized as follows. In Section 2, we outline the proposed DSC-based multispectral image compression system. In Section 3, we describe the strategy of side information generation by means of region-based adaptive prediction algorithm. In Section 4, we report the experimental results to demonstrate the performance of the proposed scheme. Section 5 concludes this paper with a summary.

\section{THE PROPOSED SYSTEM}

Based on the DSC theory, each band of multispectral images is encoded separately and the compressed streams are transmitted to the decoder for joint decoding. In this research, the first band is encoded by a conventional intra mode codec, such as lossless JPEG [2], and other bands are encoded with the proposed DSCbased codec. Figure 1 illustrates the proposed DSC-based multispectral image compression system for the $k^{\text {th }}$ band $B_{k}$ assuming that the previous $k-1$ bands have already been decoded.

We propose an efficient way to compress the image progressively in resolution, such that the decoder can quickly obtain a lowresolution layer, from which the local spectral correlation is learned and used to generate side information for the higherresolution layer. The low-resolution layer is required to be able to 
represent well the nonstationary spectral correlation of the entire multispectral image. Since spatially adjacent pixels representing the same object are very much likely to have similar spectral dependency, partial data scattered over the image acquired by sampling are chosen as a low-resolution layer in this paper.

As illustrated in Figure 1, at the encoder the image $B_{k}$ is first partitioned into a number of slices $B_{k}^{1} B_{k}^{2} \cdots B_{k}^{16}$ by sampling and each slice is encoded in the same bit-plane-by-bit-plane fashion using a bit-plane extractor and a binary LDPC encoder. The generated syndrome bits of these slices are transmitted progressively. Since spectral correlation estimation at the decoder requires basic information of $B_{k}$, some statistics of the first slice are calculated and transmitted to the decoder. At the decoder, all slices are decoded successively starting from the first slice $B_{k}^{1}$. With the knowledge of $B_{k-1}^{1}$ denoting the first slice of the adjacent band $B_{k-1}$ decoded previously and the statistics of $B_{k}^{1}$ transmitted from the encoder, a linear predictor is estimated and used to generate side information of $B_{k}^{1}$, denoted by $S I_{k}^{1}$. $B_{k}^{1}$ is then decoded using an LDPC decoder by combining $S I_{k}^{1}$ with the received syndrome bits. Different from $B_{k}^{1}$, other slices $\left\{B_{k}^{l} \mid 2 \leq l \leq 16\right\}$ are decoded in sequence with side information acquired in a distinct way because of the availability of $B_{k}^{1} B_{k}^{2} \cdots B_{k}^{l-1}$. First, the local spectral correlation is captured and used to generate side information of $B_{k}^{l}$ by means of an adaptive region-based prediction algorithm to exploit nonstationary spectral correlation. Afterwards, the statistic of correlated noise is estimated by context modeling to exploit residual correlation. The decoding performance of these slices is improved gradually by capturing more accurate spectral correlation from increasing slices. After all slices are decoded, they are regrouped to losslessly reconstruct the original image.

The following gives the verbose descriptions of detailed steps.

The partition of $B_{k}$ by sampling is performed in this fashion: $B_{k}$ is divided into $4 \times 4$ size blocks, each of which is labeled as illustrated in Figure 2. Then all pixels labeled by $l$, one pixel from each block, are grouped together to form the $l^{\text {th }}$ slice $B_{k}^{l}(l=1,2, \ldots, 16)$. These slices are appropriately ordered such that the sub-image reconstructed from $B_{k}^{1} B_{k}^{2} \cdots B_{k}^{l}$ is a sub-sampled version of the original image and is expected to be able to characterize the spectral correlation of the whole image.

Bit-planes of each slice are extracted and encoded independently using a binary LDPC encoder, generating syndrome bits $\boldsymbol{s}=\boldsymbol{x} \boldsymbol{H}$, where $\boldsymbol{H}$ is a sparse parity check matrix and $\boldsymbol{x}$ is a bit-plane. The accumulated syndrome bits are stored in a buffer and transmitted increasingly upon request. The transmission starts from the first slice and repeats for each slice, from the most significant bit-plane (MSBP) to the least significant bit-plane (LSBP).

The four components of the encoder are all lightweight in computational complexity. Firstly, sampling and extracting bit-planes involves no computation. Secondly, LDPC encoding can be implemented by energy efficient modulus 2 additions in linear complexity because of the sparseness of $\boldsymbol{H}$. Thirdly, statistics calculation leads to only relatively small increase in encoder complexity since only the first slices of two consecutive bands are involved. Therefore, the proposed scheme indeed provides a very low complexity encoder.

\begin{tabular}{|c|c|c|c|}
\hline 2 & 14 & 4 & 15 \\
\hline 12 & 5 & 9 & 6 \\
\hline 3 & 10 & 1 & 11 \\
\hline 16 & 7 & 13 & 8 \\
\hline
\end{tabular}

\section{Figure 2: Block labeling}

$B_{k}^{1}$ is decoded first. Side information of $B_{k}^{1}$ is generated by employing a $1^{\text {st }}$-order linear model:

$$
S I_{k}^{1}=\alpha_{k}^{1} B_{k-1}^{1} \quad \beta_{k}^{1}
$$

where $\alpha_{k}^{1}$ and $\beta_{k}^{1}$ characterizing the spectral correlation of $B_{k}^{1}$ and $B_{k-1}^{1}$ are obtained by Least-Square (LS) technique to minimize the mean squared prediction errors (MSPE):

$$
\begin{aligned}
& \alpha_{k}^{1}=\frac{E\left[B_{k}^{1} B_{k-1}^{1}\right]-E\left[B_{k}^{1}\right] \cdot E\left[B_{k-1}^{1}\right]}{E\left[\left(B_{k-1}^{1}\right)^{2}\right]-E\left[B_{k-1}^{1}\right] \cdot E\left[B_{k-1}^{1}\right]} \\
& \beta_{k}^{1}=E\left[B_{k}^{1}\right] \quad \alpha_{k}^{1} E\left[B_{k-1}^{1}\right]
\end{aligned}
$$

where $E[\cdot]$ represents the expectation. Except $E\left[B_{k}^{1}\right], E\left[B_{k}^{1} B_{k-1}^{1}\right]$ that are transmitted from the encoder, all other calculations involved in predictor estimation given by (2) are carried out at the decoder. $S I_{k}^{1}$ is then combined with the received syndrome bits to perform LDPC decoding under the assumption that the prediction errors are Laplace distributed.

Notice that this scheme uses only one predictor defined by (1) and only one band to predict the first slice in order to avoid additional header information and computation at the encoder. However, a single predictor is likely inadequate to cope with the spatially varying characteristics of multispectral data, and cause degradation of the compression performance for the first slice.

Unlike the first slice, other slices are decoded more efficiently by means of sophisticated spectral decorrelation method. Once the first slice is decoded, the decoder obtains a low-resolution image. By accessing the low-resolution image, the decoder is able to estimate the local spectral correlation of the current band and several previously decoded bands to generate the side information for decoding the next slice. As one more slice is decoded, a higherresolution layer is then reconstructed and used to acquire more accurate spectral correlation and higher quality side information for decoding the subsequent slice. This process is iterated until the whole image is losslessly reconstructed. Details of the side information generation algorithm for slices $\left\{B_{k}^{l} \mid 2 \leq l \leq 16\right\}$ will be discussed in Section 3.

Each slice is decoded in a similar way using an LDPC decoder after the corresponding side information is produced. Bit-planes of each slice are decoded successively starting from MSBP to LSBP, even though they are encoded independently. When decoding the less significant bit-planes, the previously decoded more significant bit-planes are used as secondary side information. For the $j^{\text {th }}$ bit-plane of the $l^{\text {th }}$ slice, the posterior probability of the $n^{\text {th }}$ bit can be computed by:

$$
p\left(x_{n}=b \mid S I_{k, n}^{l}\right) \quad \sum_{B_{k, n}^{l} \in \Gamma_{b}} p\left(B_{k, n}^{l} \mid S I_{k, n}^{l}\right), \quad b \in\{0,1\}
$$

where $x_{\mathrm{n}}$ is the $n^{\text {th }}$ bit of the current bit-plane, $B_{k, n}^{l}$ is the $n^{\text {th }}$ pixel of the $l^{\text {th }}$ slice, $S I_{k, n}^{l}$ is the side information of $B_{k, n}^{l}, \quad \Gamma_{b}$ are a set of 
values whose $j-1$ more significant bits are previously decoded, the $j^{\text {th }}$ bit is $b$ and the less significant bits are uncertain, and $p\left(B_{k, n}^{l} \mid S I_{k, n}^{l}\right)$ relates the correlation between $B_{k, n}^{l}$ and $S I_{k, n}^{l}$, which is usually assumed to be a Laplace distribution.

For each bit-plane, the decoder attempts to perform decoding upon receiving a subset of syndrome bits. If it fails, additional syndrome bits are requested from the encoder buffer through feedback. The process of request and decoding is repeated until the decoding is successful [10]. The decoder, combined with feedback, provides rate flexibility for each bit-plane, which is essential in adapting to the nonstationary characteristic of multispectral images. The estimation of such characteristic is difficult and expensive to track at the encoder. The research on rate allocation at encoder when feedback is unavailable remains as our future work.

There are two modes for incremental decoding of the image: resolution and precision. The resolution mode yields progressive spatial resolution as more slices are decoded. The precision mode successively increases pixel accuracy of the decoding slice as more bit-planes are decoded. By resolution mode, the syndrome bits are transmitted in such an order that all bit-planes of the lowest-resolution slice are first decoded, followed by all bit-planes of the next higher-resolution slice, until all slices are decoded to recover the original image in a lossless fashion. For each slice, pixels are gradually refined to full precision in the order of increasing significance. Such successive reconstruction of image adds desirable features to the codec to provide the capability for image browsing and progressive transmission, as well as natural prioritization for layered protection schemes. In addition, with the incremental slices available at the decoder, the acquired spectral correlation can be refined gradually and thus the decoding performance is enhanced, that is, the compression performance of DSC benefits from such successive decoding.

\section{SIDE INFORMATION GENERATION}

Since multispectral images are captured by observing the same area of the earth surface in different spectral intervals, the same object appears consistently in all bands, that is, pixels of the same object exhibit similar spectral dependency whereas different objects probably have distinct spectral dependencies. Thus, the optimal prediction can be acquired using a set of predictors, each of which characterizes one type of spectral dependency. To distinguish pixels with similar or different spectral correlation, the image can be first segmented into a number of statistically homogeneous regions. Pixels in the same region are assumed to exhibit similar spectral dependency.

\subsection{Image segmentation}

Although the current band is unavailable at the decoder, segmentation can be implemented on the previously decoded bands since the structures of multispectral bands are assumed to be highly correlated. The objective of segmentation is to single out regions with pixels of each region having similar spectral correlation. Therefore, similarity measurement of regions should depend not only on the spatial statistic but also on the spectral features. In our research, the image is initially segmented by a k-means algorithm [11] using a vector spanning the spectral dimension, denoted by $v_{n}=\left\{B_{k-i, n} \mid i \quad 1, \cdots, m\right\}$, as the feature of the $n^{\text {th }}$ pixel. This cluster- ing algorithm results in over-segmentation with a large number of small regions and even isolated points since spatial correlation is not considered. To acquire textural regions, a MRF model over region adjacency graph (RAG) of the preliminary segmented regions is defined and over-segmented regions are appropriately merged to minimize the defined energy function [12]. Different from the conventional region-based entropy coding schemes [13], there is no need to transmit the segmentation map since the segmentation is implemented at the decoder. Therefore, the image can be elaborately segmented into a number of relatively small and moderate regions such that each region exhibits homogeneous statistics.

\subsection{Adaptive Region-based Prediction}

For the $l^{\text {th }}$ slice $B_{k}^{l}(l=2,3, \ldots, 16)$, the side information is generated by a set of linear predictors representing the local spectral correlation of the current band and a number of previously decoded bands. Based on the knowledge of $l-1$ slices of the current band and $m$ previous bands $\left\{B_{k-i} \mid i=1,2, \cdots, m, m<k\right\}$ that have been decoded, the spatially varying spectral correlation is captured. To better adapt to the nonstationary characteristics of multispectral images, a particular predictor is adaptively estimated for each pixel of $B_{k}^{l}$ by learning spectral correlation from neighboring pixels. Denote $B_{k, n}^{l}$ the $n^{\text {th }}$ pixel of $B_{k}^{l}$ and $\left(v_{n}, h_{n}\right)$ the corresponding coordinate. Define a 2-D neighborhood for $B_{k, n}^{l}$ by

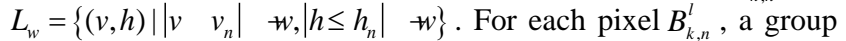
of neighboring pixels that have already been decoded are selected as follows and used to estimate the local spectral correlation:

1) Pixels belonging to the same region with $B_{k, n}^{l}$ are selected with top-priority. Denote the number of the selected pixels $c^{\prime}$. Neighboring decoded pixels in the same region are picked starting from the nearest one until $c^{\prime}=c$, where $c$ is a predefined value representing the number of pixels that are expected to be selected. For some small regions, there are very few or even no decoded pixels, i.e., $c^{\prime}<c$. That is, neighboring decoded pixels in the same region with $B_{k, n}^{l}$ are not enough to represent the local statistic of $B_{k, n}^{l}$. If so, go to step 2).

2) Neighboring pixels with region labels different from $B_{k, n}^{l}$ are used as secondary candidate. The difference between the neighboring pixel $B_{k, v, h}$ at position $(v, h)$ and $B_{k, n}^{l}$ is computed by:

$$
d=\sum_{i=1}^{m}\left(B_{k-i, n}^{l} \quad B_{k-i, v, h}\right)^{2}
$$

where $(v, h) \in L_{w}$. If $d$ is less than a preset threshold, the pixel $B_{k, v, h}$ is selected. If there still exists $c^{\prime}<c$ after all pixels in $L_{w}$ are visited, go to step 3).

3) Other decoded pixels in $L_{w}$ are selected without any constraint until $c^{\prime}=c$.

The eligible pixels are visited in an increasing order of the spatial distance from $B_{k, n}^{l}$ in all the three above steps. Since the selected pixels are likely inadequate to estimate an $m^{\text {th }}$-order predictor, $m$ 1 st -order predictors are adopted:

$$
S I_{k, n}^{l(i)}=\alpha_{k-i, n} B_{k-i, n}^{l} \quad \beta_{k-i, n}, \quad i \neq 1,2, \cdots, m
$$


where $\alpha_{k-i, n}, \beta_{k-i, n}$ represent the spectral correlation of the current band $B_{k}$ and the previous band $B_{k-i}(\mathrm{i}=1,2, \ldots m)$ at the position of $\left(v_{n}, h_{n}\right)$. The coefficients of each predictor are calculated using the selected pixels by means of LS algorithm to yield minimum MSPE, denoted by $m s p e_{k-i, n}^{l} . B_{k, n}^{l}$ is then predicted as the weighted sum of the output of $m$ predictors:

$$
S I_{k, n}^{l}=\sum_{i=1}^{m} u_{k-i, n}^{l} S I_{k, n}^{l(i)}
$$

where

$$
u_{k-i, n}^{l}=\frac{1}{\sum_{j=1}^{m} \frac{m s p e_{k-i, n}^{l}}{m s p e_{k-j, n}^{l}}}
$$

This region-based prediction algorithm captures the local spectral features by actually exploiting both spectral and spatial statistics. Thus, it is quite efficient for multispectral data decorrelation.

\subsection{Correlated Noise Statistic Estimation}

Although it is assumed to generate a memoryless version of prediction errors by performing data decorrelation, the prediction errors (also called correlated noise in DSC) still exhibit residual correlation. To exploit this characteristic, context modeling is carried out to partition the noise into several statistically homogeneous signals. Since noise values are unknown as the original image is unavailable, the context of each pixel $B_{k, n}^{l}$ is computed as the MSPE of the selected neighboring pixels:

$$
c t x_{k, n}^{l}=\sum_{i=1}^{m} u_{k-i, n}^{l} m s p e_{k-i, n}^{l}
$$

The noise is then classified into a number of classes by partitioning the histogram of the context. As a consequence, multiple models, each following a Laplace distribution but with different variance, will better approximate the spatially varying statistics, and thus improve the decoding performance.

\section{EXPERIMENTAL RESULTS}

The proposed scheme has been implemented on several Landsat Thematic Mapper (TM) images, with $256 \times 256$ pixels, 8 bits/pixel and six bands (the sixth band was omitted because of its poor resolution and limited spectral correlation). Figure 3 shows the fifth band of two test data sets. The first one represents an area near Lisbon and the second one represents an area in the southwest of Jiangsu, China. To achieve optimal decorrelation, the bands sequence is rearranged to maximize the average crosscorrelation between any couple of consecutive bands. The optimum ordering for TM images was acquired offline to be $1 \rightarrow 2 \rightarrow 3 \rightarrow 7 \rightarrow 5 \rightarrow 4$ [5]. Assuming that the first band is available at the decoder, the other bands are compressed by the proposed DSC codec. All previously decoded bands $(m=k-1)$ are used to predict the current band. The LDPC code is regular with degree 3 and length 4096 bits so as to encode a bit-plane of each slice as one code.

\subsection{Compression Results}

The variance of prediction errors are reported in Table 1 and Table 2, where $\sigma_{l}^{2}$ represents the MSPE of the $l^{\text {th }}$ slice produced by our region-based prediction algorithm and $\sigma^{2}$ represents the MSPE of the whole image produced by the classified 3-D prediction algorithm proposed in [6] with 12 predictors and $22^{\text {nd }}$-order $3-D$ causal prediction. The number of selected neighboring pixels $c$ is set to be the number of decoded pixels in a $L_{\mathrm{w}}$ neighborhood with $w=2$ for slices $B_{k}^{l}(l \leq 4)$ and $w=1$ for slices $B_{k}^{l}(l>4)$ assuming that all relative pixels are in a homogeneous region. For actual neighboring pixels selection, $w$ is set to be 5 to guarantee a high probability that there are enough decoded pixels with the same label in the $L_{w}$ neighborhood. As observed from Table 1 and Table $2, \sigma_{l}^{2}$ almost diminish monotonously with respect to the increase of $l$. This indicates that more accurate spectral correlation is acquired as more data are available. Comparing with the classified 3-D prediction algorithm, the proposed prediction algorithm produces poorer results for slices $B_{k}^{l}$ ( $l \leq 4$ for TM-Lisbon, $l \leq 8$ for TM-Jiangsu) because the data used to estimate the spectral correlation are too sparse and include no decoding pixels. However, the prediction precision is improved for slices $B_{k}^{l}$ ( $l>4$ for TM-Lisbon, $l>8$ for TM-Jiangsu) since with enough data available, the region-based prediction algorithm better adapts to the spatially varying characteristic of multispectral data, which outweighs the drawback in the lack of the decoding pixels.

The bit-rates per pixel are reported in Table 3 and Table 4. The performance of the proposed scheme is compared with that of the state-of-the-art lossless compression techniques. The lossless JPEG standard [2] is selected as a typical 2-D compression scheme with low encoding complexity. The 3-D differential pulse code modulation (DPCM) based scheme proposed in [6], the best performance scheme to our knowledge, is used as the benchmark for performance evaluation. As shown in Table 3 and Table 4, the DSC scheme significantly outperforms the lossless JPEG codec by $0.7 \sim 1.6$ bits per pixel (bpp). Compared with the 3-D DPCM based scheme, equivalent and even lower bit-rates are obtained for TM-Lisbon while a loss of about $0.2 \mathrm{bpp}$ is observed for TMJiangsu. The compression efficiency is significantly affected by the prediction performance, which can be observed from the tables. Better prediction performance together with much less header information contributes to the improved compression efficiency for some bands of TM-Lisbon.

The proposed region-based prediction algorithm is different in three aspects with respect to the classified 3-D prediction algorithm proposed in [6]. First, the region-based prediction algorithm can better adapt to the nonstationary characteristic of the image than the block-based prediction algorithm adopted in [6]. Therefore, the proposed scheme can improve the prediction performance significantly for images with weaker stationarity, such as TM-Lisbon. Second, having only partial access to the image, our prediction algorithm is based on the assumption that pixels in the same region retain similar spectral dependency. Hence, the scheme is able to perform well in learning the local spectral correlation from neighboring pixels for TM-Lisbon, mostly consisting of smooth regions, but not so well for TM- Jiangsu, mainly consisting of texture regions, which is probably less consistent with the consumption. Third, no spatial prediction is adopted in our scheme.

\subsection{Encoding complexity}

The motivation of the proposed DSC scheme is to design a low complexity encoder. To illustrate the encoder complexity reduction for the proposed scheme, Table 5 depicts the encoding time 
per band, averaged over the test data sets of 100 runs, using different encoders run on a computer with a Pentium IV $3 \mathrm{GHz}$ CPU. As expected, the DSC encoder reveals remarkable complexity reduction compared with 3-D DPCM [6] and even weighs lighter than lossless JPEG [2]. Therefore, the proposed DSC scheme indeed results in a lightweight encoder with high compression efficiency suitable for onboard applications.

\section{CONCLUSION}

In this paper, we have described a novel DSC scheme for multispectral images in order to design a lightweight yet efficient encoder by moving data decorrelation operations to the decoder. The image is compressed progressively so that the decoder can have partial access to the image initially, from which the local spectral correlation is learned and used to generate the side information for decoding the next resolution level. As more slices are decoded, higher-resolution image is reconstructed and more accurate spectral correlation is acquired, resulting in gradually improved decoding performance. The major innovation lies in the design of adaptive region-based prediction algorithm to capture both spatial and spectral varying characteristic of multispectral images. The experimental results demonstrate that the proposed scheme yields excellent compression performance, and is competitive with the best algorithms available in literature with very low complexity encoder suitable for onboard processing in multispectral image acquisition.

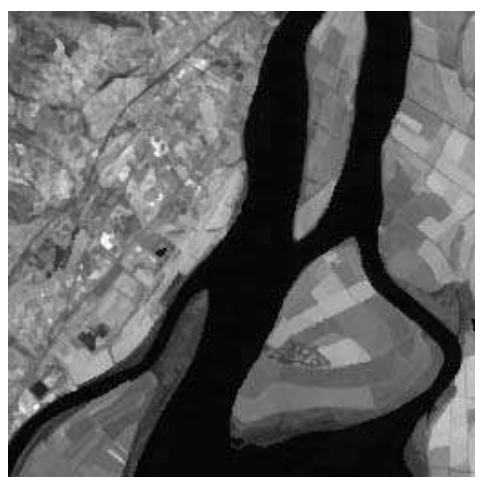

(a) TM-Lisbon

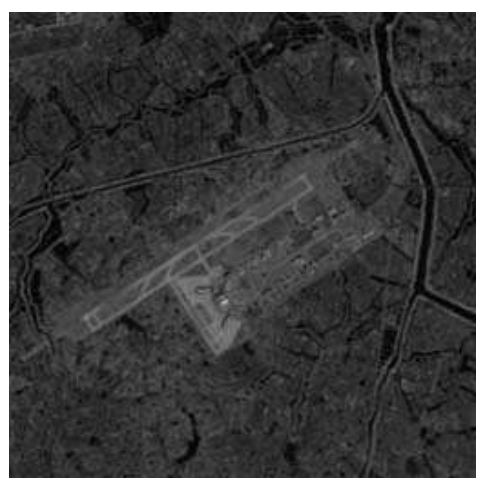

(b) TM-Jiangsu

Figure 3: The fifth band of the test images
Table 1. Variance of prediction errors of TM-Lisbon

\begin{tabular}{|c|c|c|c|c|c|c|}
\hline & $\boldsymbol{c}$ & Band2 & Band3 & Band7 & Band5 & Band4 \\
\hline$\sigma_{1}{ }^{2}$ & & 5.38 & 39.62 & 108.34 & 209.98 & 252.28 \\
\hline$\sigma_{2}{ }^{2}$ & 4 & 1.70 & 8.03 & 17.12 & 34.55 & 59.15 \\
\hline$\sigma_{3}{ }^{2}$ & 4 & 1.67 & 6.92 & 11.94 & 25.43 & 36.74 \\
\hline$\sigma_{4}{ }^{2}$ & 4 & 1.44 & 6.11 & 11.86 & 21.76 & 33.11 \\
\hline$\sigma_{5}{ }^{2}$ & 4 & 0.59 & 2.96 & 4.33 & 7.26 & 12.10 \\
\hline$\sigma_{6}{ }^{2}$ & 4 & 0.55 & 2.10 & 3.17 & 5.93 & 11.71 \\
\hline$\sigma_{7}{ }^{2}$ & 4 & 0.52 & 1.67 & 3.17 & 5.76 & 10.92 \\
\hline$\sigma_{8}{ }^{2}$ & 4 & 0.54 & 1.71 & 2.63 & 5.65 & 8.56 \\
\hline$\sigma_{9}{ }^{2}$ & 4 & 0.44 & 1.86 & 2.65 & 4.41 & 6.67 \\
\hline$\sigma_{10}{ }^{2}$ & 5 & 0.42 & 1.32 & 1.86 & 3.90 & 6.05 \\
\hline$\sigma_{11}{ }^{2}$ & 5 & 0.44 & 1.25 & 2.08 & 4.17 & 6.36 \\
\hline$\sigma_{12}{ }^{2}$ & 6 & 0.40 & 1.23 & 2.14 & 4.20 & 6.08 \\
\hline$\sigma_{13}{ }^{2}$ & 6 & 0.42 & 1.43 & 2.17 & 3.74 & 5.91 \\
\hline$\sigma_{14}{ }^{2}$ & 7 & 0.39 & 1.09 & 2.10 & 3.91 & 5.79 \\
\hline$\sigma_{15}{ }^{2}$ & 7 & 0.42 & 1.14 & 2.05 & 4.02 & 5.56 \\
\hline$\sigma_{16}{ }^{2}$ & 8 & 0.39 & 1.17 & 1.97 & 3.86 & 5.52 \\
\hline$\sigma^{2}$ & & 0.72 & 2.13 & 4.91 & 9.40 & 11.17 \\
\hline & & & & & & \\
\hline
\end{tabular}

Table 2. Variance of prediction errors of TM-Jiangsu

\begin{tabular}{|c|c|c|c|c|c|c|}
\hline & $\boldsymbol{c}$ & Band2 & Band3 & Band7 & Band5 & Band4 \\
\hline$\sigma_{1}{ }^{2}$ & & 1.90 & 7.92 & 35.54 & 34.83 & 18.28 \\
\hline$\sigma_{2}{ }^{2}$ & 4 & 1.89 & 6.10 & 19.29 & 18.31 & 12.24 \\
\hline$\sigma_{3}{ }^{2}$ & 4 & 1.72 & 5.52 & 16.97 & 16.51 & 10.12 \\
\hline$\sigma_{4}{ }^{2}$ & 4 & 1.64 & 5.44 & 16.12 & 16.49 & 11.62 \\
\hline$\sigma_{5}{ }^{2}$ & 4 & 1.31 & 4.08 & 11.53 & 12.55 & 8.05 \\
\hline$\sigma_{6}{ }^{2}$ & 4 & 1.32 & 4.12 & 11.32 & 12.79 & 8.04 \\
\hline$\sigma_{7}{ }^{2}$ & 4 & 1.36 & 4.19 & 10.93 & 12.23 & 7.98 \\
\hline$\sigma_{8}{ }^{2}$ & 4 & 1.35 & 4.05 & 10.59 & 11.86 & 7.16 \\
\hline$\sigma_{9}{ }^{2}$ & 4 & 0.91 & 2.44 & 6.23 & 8.27 & 4.41 \\
\hline$\sigma_{10}{ }^{2}$ & 5 & 0.89 & 2.32 & 6.15 & 8.13 & 4.35 \\
\hline$\sigma_{11}{ }^{2}$ & 5 & 0.83 & 2.27 & 6.12 & 8.08 & 4.16 \\
\hline$\sigma_{12}{ }^{2}$ & 6 & 0.87 & 2.25 & 6.13 & 8.10 & 4.25 \\
\hline$\sigma_{13}{ }^{2}$ & 6 & 0.84 & 2.18 & 6.20 & 8.13 & 4.04 \\
\hline$\sigma_{14}{ }^{2}$ & 7 & 0.86 & 2.21 & 6.14 & 8.04 & 4.11 \\
\hline$\sigma_{15}{ }^{2}$ & 7 & 0.81 & 2.12 & 6.12 & 7.92 & 4.14 \\
\hline$\sigma_{16}{ }^{2}$ & 8 & 0.83 & 2.08 & 6.07 & 7.83 & 4.07 \\
\hline$\sigma^{2}$ & & 0.95 & 2.44 & 6.51 & 8.34 & 4.53 \\
\hline & & & & & & \\
\hline
\end{tabular}


Table 3. Bit-rates per pixel of TM-Lisbon (bpp)

\begin{tabular}{|c|c|c|c|}
\hline Codec & Lossless JPEG & DSC & 3-D DPCM \\
\hline Band2 & 3.50 & 1.84 & 1.75 \\
\hline Band3 & 4.04 & 2.63 & 2.58 \\
\hline Band7 & 3.93 & 2.78 & 2.82 \\
\hline Band5 & 4.43 & 3.14 & 3.25 \\
\hline Band4 & 4.04 & 3.23 & 3.33 \\
\hline
\end{tabular}

Table 4. Bit-rates per pixel of TM-Jiangsu (bpp)

\begin{tabular}{|c|c|c|c|}
\hline Codec & Lossless JPEG & DSC & 3-D DPCM \\
\hline Band2 & 3.60 & 2.55 & 2.36 \\
\hline Band3 & 4.39 & 3.38 & 3.12 \\
\hline Band7 & 4.76 & 4.01 & 3.76 \\
\hline Band5 & 5.48 & 4.21 & 3.99 \\
\hline Band4 & 4.75 & 3.76 & 3.52 \\
\hline
\end{tabular}

Table 5. Average encoding time per band (ms)

\begin{tabular}{|c|c|c|c|}
\hline Encoder & Lossless JPEG & DSC & 3-D DPCM \\
\hline Time & 62 & 31 & 34476 \\
\hline
\end{tabular}

\section{ACKNOWLEDGMENTS}

This work is supported by NSFC General Program under contract No. 60672161 and NSFC Key Program under contract No. 60736043 and No. 60632040.

\section{REFERENCES}

[1] Taubman, D., and Marcellin, M. W., JPEG 2000, Image compression, Fundamentals, Standards and Practice. Norwell, MA: Kluwer, 2002.

[2] Pennebaker, W.B., and Mitchell, J.L., JPEG: Still Image Compression Standard, New York: Van Nostrand Reinhold, 1993.

[3] Penna, B., Tillo, T., Magli, E., and Olmo, G., "Progressive 3D coding of hyperspectral images based on JPEG 2000,"
IEEE Geosci. Remote Sensing Letter, vol. 3, pp. 125-129, Jan. 2006.

[4] Benazza-Benyahia, A., Pesquet, J.-C., and Hamdi, M., "Vector-lifting schemes for lossless coding and progressive archival of multispectral images," IEEE Trans. Geosci. Remote Sensing, vol. 40, pp. 2011-2024, Sept. 2002.

[5] Aiazzi, B., Alba, P., Alparone, L., and Baronti, S., "Lossless Compression of Multi/hyperspectral Imagery based on a 3-D Fuzzy Prediction," IEEE Trans. Geosci. Remote Sensing, vol. 37, pp. 2287-2294, Sept. 1999.

[6] Aiazzi, B., Alparone, L., and Baronti, S. "Near-Lossless Compression of 3-D Optical Data," IEEE Trans. Geosci. Remote Sensing, vol. 39, pp. 2547-2557, Nov. 2001.

[7] Slepian, D., and Wolf, J.K., "Noiseless Coding of Correlated Information Sources," IEEE Trans. Inform. Theory, vol. 19, pp. 471-480, July 1973.

[8] Cheung, N.-M., Tang, C., Ortega, A., and Raghavendra, C.S., "Efficient Wavelet-based Predictive Slepian-Wolf Coding for Hyperspectral Imagery," EURASIP Journal on Signal Processing, vol. 86(11), pp. 3180-3195, 2006

[9] Magli, E., Barni, M., Abrardo, A., and Grangetto, M., "Distributed Source Coding Techniques for Lossless Compression of Hyperspectral Images," EURASIP Journal on Advances in Signal Processing, vol. 2007, 2007.

[10] Varodayan, D., Aaron A., and Girod, B., "Rate-Adaptive Codes for Distributed Source Coding," EURASIP Signal Processing: Image Communication, vol.21, pp.462-475, July 2006.

[11] Kanungo, T., Mount, D. M., Netanyahu, N. S., Piatko, C. D., and Wu, A. Y., "An Efficient k-Means Clustering Algorithm: Analysis and Implementation," IEEE Trans. Pattern Analysis and Machine Intelligence, vol. 24, pp. 881-892, July 2002.

[12] Sarkar, A., Biswas, M. K., Kartikeyan, B., Kumar, V., Majumder, K. L., and Pal, D. K., "A MRF Model-based Segmentation Approach to Classification for Multispectral Imagery," IEEE Trans. Geosci. Remote Sensing, vol. 40, pp. 1102-1113, May 2002.

[13] Cagnazzo, M., Poggi, G., and Verdoliva, L., "Region-Based Transform Coding of Multispectral Images," IEEE Trans. Image Processing, vol. 16, pp. 2916-2926, Dec. 2007. 